\title{
Prevalencia de etiologías del Síndrome de Dificultad Respiratoria del Recién Nacido. Perfil materno y neonatal en centro Neonatal
}

\author{
Prevalence of Newborn Respiratory Distress Syndrome etiologies. \\ Maternal and neonatal profile in the Neonatal Center
} Marcela Díaz Navarrete ${ }^{1}$, Carolina Ramírez Álvarez ${ }^{2}$, Fernanda Vergara Berríos ${ }^{3}$.

${ }^{1}$ Matrona, Directora Dpto. de Promoción de la Salud de la Mujer y el Recién Nacido. Universidad de Chile. Santiago, Chile. ${ }^{2}$ Matrona. Dpto. de Promoción de la Salud de la Mujer y el Recién Nacido. Universidad de Chile. Santiago, Chile. ${ }^{3}$ Matrona. Centro de Salud Familiar El Manzano. Servicio de Salud Metropolitano.

Santiago. Chile.

*Autor para correspondencia: mdiaz@uchile.com RECIBIDO: 27 de Mayo de 2020 APROBADO: 11 de Agosto de 2000

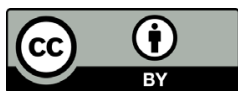

DOI: $10.22370 /$ rev.mat.1.2020.2247

\section{LOS AUTORES DECLARAN NO TENER CONFLICTO DE INTERESES}

Palabras claves: Síndrome de Dificultad Respiratoria, etiologías, estudio de prevalencia.

Key words: Respiratory Distress Syndrome, etiologies, prevalence study.

\section{RESUMEN}

Antecedentes: el Síndrome de Dificultad Respiratoria (SDR) en neonatos, es causado por variadas etiologías y tiene una prevalencia histórica de $1 \%$. No existen datos actualizados respecto a la prevalencia del SDR en Chile ni el porcentaje atribuible a cada etiología.

Objetivo: determinar la prevalencia de las etiologías del SDR y las características maternas y neonatales de los recién nacidos (RN) que cursaron SDR en un Hospital de Alta Complejidad Neonatal, año 2014.

Material y método: estudio descriptivo de prevalencia. Se analizaron fichas clínicas de RN que cursaron SDR. Se trabajó con el universo de los RN equivalente a 141.

Resultados: la prevalencia del SDR correspondió al 2,7\%, siendo la Taquipnea Transitoria la etiología más frecuente $(58,2 \%)$. Dentro de las características maternas destaca la edad con una mediana de 27 años; chilenas $72,3 \%$, dueña de casa $38,3 \%$ y estado nutricional sobrepeso y obesidad en un $58,6 \%$.
El tipo de parto más frecuente fue la cesárea con un $69,5 \%$. Las características neonatales más relevantes son el sexo masculino, edad gestacional entre $34 \mathrm{y}$ 36 semanas y una mediana de 17 días de hospitalización. La principal complicación asociada fue Ductus Arteriosus Persistente y resolución del cuadro fue $62,4 \%$ neonato de alta sano versus un $27,6 \%$ con alguna patología. El síndrome se asocia a una letalidad de $3,2 \%$ siendo la principal etiología involucrada la Enfermedad de Membrana Hialina.

Conclusión: existe casi una triplicación en la prevalencia del SDR, siendo la etiología más frecuente la Taquipnea Transitoria.

\section{ABSTRACT}

Background: Respiratory Distress Syndrome (RDS) of the newborn is caused by various etiologies and has an historical prevalence of $1 \%$. Nevertheless there is no current data on either the SDR prevalence in Chile or 
Prevalencia de etiologías del Síndrome de Dificultad Respiratoria del Recién Nacido. Perfil materno y neonatal en centro Neonatal \begin{tabular}{l|l} 
[Mat. Actual. 2020] Nro. 1: 07-16 & ISSN: $2452-5820$ \\
DOI: $10.22370 /$ rev.mat.1.2020.2247
\end{tabular} the percentage attributable to each etiology nor characterizing the neonatal symptoms and the maternal characteristics.

Objective: to determine the prevalence of RDS etiologies plus the maternal and neonatal features of the newborns hospitalized whit the diagnosis RDS in a Hospital with High Neonatal Complexity during 2014.

Methods: descriptive study of prevalence. The medical records of prevalence RN who studied with RDS were analyzed. We worked with the universe equivalent to $141 \mathrm{RN}$.

Results: the prevalence of SDR corresponds to $2,7 \%$, the Transient tachypnea most common cause with $58,2 \%$. Inside features include maternal age with a median of 27 years; Chilean $72,3 \%, 38,3 \%$ housewife and nutritional status overweight and obesity in $58,6 \%$. The most common type of delivery is cesarean $69,5 \%$. The most important features are neonatal males, gestational age between 34 and 36 weeks and a median of 17 days of hospitalization. The main complication was associated Ductus Arteriosus Persistent and resolution of syndrome was 62,4\% healthy newborn discharge versus $27,6 \%$ with some pathology. The syndrome is associated with a death rate of $3,2 \%$ is the main etiology involved Hyaline Membrane Disease.

Conclusion: there is almost a threefold increase in the prevalence of RDS, the most common cause Transient tachypnea.

\section{INTRODUCCION}

La transición a la vida extrauterina está determinada por una serie de cambios fisiológicos, los cuales involucran complejos mecanismos de homeostasis y maduración de sistemas (1), los más importantes se producen a nivel respiratorio y circulatorio, representando un período de mayor vulnerabilidad y en donde existen más probabilidades de presentar complicaciones, enfermar o morir (1).

Entre las complicaciones más frecuentes está el Síndrome de Dificultad Respiratoria (SDR). Este se caracteriza por presentar: taquipnea, cianosis, quejido, retracción subcostal y grados variables de compromiso de la oxigenación neonatal (2), teniendo variadas etiologías, siendo las de mayor frecuencia la Enfermedad de Membrana Hialina (EMH), Bronconeumonía (BNM), Síndrome Aspirativo Meconial (SAM) y Taquipnea Transitoria (TT) y en una menor frecuencia malformaciones de las vías aéreas y pulmonares, presencia de rupturas alveolares con $\mathrm{Neu}^{-}$ motórax, Hernia Diafragmática Congénita (HDC), Hipertensión Arterial Pulmonar (HAP) y otros trastornos como son la hipoglicemia, acidosis metabólica, anemia severa, poliglobulia, alteraciones del sistema nervioso central, cardiopatía congénita, entre otras (3).

La mortalidad en recién nacidos (RN) vivos por causas respiratorias no asociada a prematurez, $\mathrm{CO}^{-}$ rresponde entre un $10 \%$ a $15 \%$ de la mortalidad neonatal, es decir, entre 120 a 180 muertes por año (2). Además, las afecciones respiratorias conllevan variadas complicaciones tales como, la ruptura alveolar con neumotórax o neumomediastino, septicemia, hemorragia pulmonar, apnea, hemorragia intracraneana, Displasia Broncopulmonar, Retinopatía del Prematuro, entre otras (4).

Según cifras aportadas por The National Neonatal Perinatal Database Network, cerca del 6\% al 10\% de los neonatos sufren enfermedad respiratoria a causa de neumonía, Síndrome Aspirativo Meconial, Síndrome de Dificultad Respiratoria y Apnea (5). En Chile según estadísticas del año 2011 entregadas por el Departamento de Estadísticas e Información de Salud, hubo un total de 248.879 recién nacidos vivos (6) y se señala que: "El SDR, con sus distintas etiologías, afecta a un 1,0 \% de los nacidos vivos" (Guía Clínica GES Sindrome de Dificultad Respiratoria en el recién nacido). Además, se estima que los niños con SDR representan alrededor de un 7 a 10\% de los ingresos a las Unidades de Cuidado Intensivo Neonatal (3).

Una de las patologías de mayor impacto en las unidades de neonatología es la Enfermedad de Membrana Hialina, causada por la ausencia o déficit de surfactante pulmonar (6). Dentro de los principales factores de riesgo es la menor edad gestacional, por lo que es necesario administrar glucocorticoides antes de las 34 semanas, disminuyendo el riesgo de EMH o su gravedad (7). Se ha observado que los neonatos pretérmino tardíos que tienen antecedente de diabetes materna, tienen mayor probabilidad de presentar EMH (8-9). Por otro lado, el nacer por cesárea sin trabajo de parto, aumenta el riesgo 3,5 veces más respecto de los neonatos que nacen por parto normal (10). 
Prevalencia de etiologías del Síndrome de Dificultad Respiratoria del Recién Nacido. Perfil materno y neonatal en centro Neonatal
[Mat. Actual. 2020] Nro. 1: 07-16 $\mid \begin{aligned} & \text { ISSN: } 2452-5820 \\ & \text { DOI: } 10.22370 / \text { rev.mat.1.2020.2247 }\end{aligned}$
La Taquipnea Transitoria, es una patología de carácter benigna, autolimitada, más frecuente en $\mathrm{RN}$ de término y pretérminos tardíos de 34 - 36 semanas (11-12) y generalmente es la causa más frecuente de SDR, está asociada a la persistencia del líquido pulmonar después de nacer (13). La cesárea sin trabajo de parto, es considerada uno de los principales factores de riesgo, ya que los estímulos hormonales fetales y neonatales tales como el cortisol, catecolaminas y vasopresinas que favorecen la reabsorción del líquido pulmonar están disminuidos (14).

La Bronconeumonía, se define como una infección del tracto respiratorio a consecuencia de infecciones connatales o infecciones nosocomiales (2), siendo factor de riesgo para esta la infección vaginal (15) y la rotura de membranas prolongada, ya que el riesgo de infección aumenta proporcionalmente con la duración del período de latencia (16), para la cual la administración de profilaxis antibiótica es fundamental (7).

El Síndrome Aspirativo Meconial, cuadro que se produce secundario a la aspiración de meconio en la vía aérea, pudiendo ocurrir antes o durante el nacimiento (2). La expulsión de meconio se da por un incremento de la peristalsis y la relajación del esfínter anal, en respuesta a un aumento del estímulo vagal durante las compresiones del cordón umbilical o durante la hipoxia, en el cual el tono simpático aumenta (17). El SAM está asociado a edades más tardías (18), registrándose una prevalencia de 0,43 por 1000 nacidos vivos, en el cual un $34 \%$ de estos son mayores de 40 semanas de edad gestacional (19). Otro factor de riesgo es la presencia de oligohidramnios, ya que aumenta la probabilidad de compresiones de cordón (20), también los partos distócicos en que se expone al feto a mayor riesgo de hipoxia anteparto e intraparto y a una disminución del flujo placentario (21). Además, la listeriosis congénita se ha asociado como factor de riesgo en recién nacidos menores de 36 semanas (11).

La Hipertensión Arterial Pulmonar, se caracteriza por un incremento de la reactividad vasoconstrictora vascular y un aumento de la capa muscular de la pared arterial pulmonar (22), lo que se traduce en una reducción del lumen vascular, aumento de la resistencia vascular pulmonar y de la presión de la arteria pulmonar por sobre los $25 \mathrm{mmHg}$ (23), favoreciendo una hipoxemia severa por persistencia de shunts de derecha a izquierda, entre otros mecanismos (24). Las causas de esta patología no son del todo conocidas, pero se asocia a varias posibles etiologías (22), asociadas a eventos como hipoxia crónica intrauterina y asfixia en un $43,7 \%$, o a complicaciones agudas como hipotermia severa e hipoglicemia; también puede ser secundario a otras etiologías del SDR, como por ejemplo, aspiración de meconio en un $50 \%$, sepsis por Estreptococo Grupo B en un $31,3 \%$ y EMH en un $18,8 \%$ (25).

Finalmente, se describe que los RN de sexo masculino que presentaron SDR corresponden a un $65,4 \%$ versus el sexo femenino en un $34,6 \%$. Además, la morbilidad y mortalidad del SDR en RN varones es más alta que en RN femeninos (26).

No existen datos actuales con respecto a la prevalencia del SDR en Chile, ni del porcentaje atribuible a cada etiología, ni estudios en los que se caracterice a los RN y sus madres según caracterización sociodemográfica propia del país. Si bien existe conocimiento de los diferentes factores y marcadores de riesgo pre y postnatales en el desarrollo del SDR, en este estudio se busca evidenciarlos, incluyendo además las características maternas y de los RN que presentan este cuadro, por lo que el objetivo de esta investigación es determinar la prevalencia de las etiologías del SDR del RN y las características maternas y neonatales de recién nacidos hospitalizados que cursaron con Síndrome de Dificultad Respiratoria en el Hospital Clínico San Borja Arriarán, Santiago Chile, durante el año 2014, contribuyendo en el conocimiento de los principales factores relacionados a las distintas etiologías del SDR, para promover el desarrollo de estrategias enfocadas en la prevención primaria en la atención de las gestantes.

\section{MATERIAL Y MÉTODO}

Tipo de estudio:

Estudio descriptivo de prevalencia. El objetivo principal fue determinar la prevalencia de SDR del $\mathrm{RN}$ y cada una de sus etiologías, caracterizar a los neonatos con SDR y a sus madres según sus características obstétricas y sociodemográficas.

\section{Sujetos de estudio:}

Se incluyó a neonatos que desarrollaron SDR, nacidos y hospitalizados en la unidad de Neonatología del Hospital San Borja Arriarán durante el año 
Prevalencia de etiologías del Síndrome de Dificultad Respiratoria del Recién Nacido. Perfil materno y neonatal en centro Neonatal \begin{tabular}{l|l} 
[Mat. Actual. 2020] Nro. 1: 07-16 & $\begin{array}{l}\text { ISSN: 2452-5820 } \\
\text { DOI: 10.22370/rev.mat.1.2020.2247 }\end{array}$
\end{tabular}
2014. En total se analizaron 141 fichas clínicas de RN y fichas perinatales maternas. Se excluyeron a $8 \mathrm{RN}$ puesto que ingresaron a la Unidad de Neonatología desde otros centros hospitalarios sin tener todos los registros necesarios para nuestro estudio. Según el Departamento de Estadísticas e Información de Salud del Hospital San Borja Arriarán, en el año 2014 hubo un total de 5.228 RN nacidos vivos.

\section{Variables:}

Dentro de las etiologías del SDR se consideró: Enfermedad de Membrana Hialina, Bronconeumonía, Síndrome Aspirativo Meconial, Taquipnea Transitoria, Hernia Diafragmática Congénita, Hipertensión Arterial Pulmonar entre otras, de acuerdo al diagnóstico médico registrado en ficha clínica según criterio diagnóstico de la Guía Clínica GES Síndrome de Dificultad Respiratoria en el recién nacido.

Las madres de los RN se caracterizaron según las siguientes variables, siendo cuantitativa edad y las variables cualitativas son las siguientes: estado civil, nivel educacional, comuna de residencia, nacionalidad, previsión de salud y ocupación, antecedentes personales de patologías maternas (diabetes, tuberculosis pulmonar, hipertensión arterial sistémica, cardiopatía, entre otras), historia obstétrica (gestas, partos y abortos), número de fetos en el embarazo actual, patologías relacionadas con la gestación (preeclampsia, eclampsia, HELLP, diabetes gestacional, infecciones urinarias, corioamnionitis, $\mathrm{co}^{-}$ lestasia intrahepática del embarazo, restricción del crecimiento intrauterino (RCIU), oligohidramnios, amenaza parto pretérmino, desproporción céfalo pélvica, anemia, rotura prematura de membranas (RPM), placenta previa y desprendimiento prematuro de placenta normo inserta). Estado nutricional (bajo peso, normal, sobrepeso, obesidad), hábitos (tabaco, alcohol y drogas). Hospitalización en Alto Riesgo, tipo de ruptura de membranas, tiempo de membranas rotas (intervalos), características del líquido amniótico, administración de corticoterapia y profilaxis antibiótica para Estreptococo Grupo B, presencia de trabajo de parto y tipo de parto.

Se caracterizó a los neonatos con SDR según las siguientes variables, siendo las cuantitativas: edad gestacional pediátrica, peso y talla, administración de corticoterapia, esquema administrado de surfactante, drogas vasoactivas y frecuencia de esquema tratamiento para Ductus Arteriosus Persistente
(DAP) y parámetros cardiorrespiratorios. Días de: tratamiento para apneas, tratamiento con antibióticos, oxigenoterapia y de hospitalización. Las variables cualitativas fueron las siguientes: sexo, Apgar, clasificación según edad gestacional y peso, necesidad de maniobras de reanimación y asfixia perinatal, momento de ocurrencia del SDR, administración de fármacos (surfactante exógeno, antibióticos, drogas vasoactivas, Sildenafil, óxido nítrico inhalatorio). Tratamiento farmacológico para apneas y DAP. Necesidad de oxigenoterapia, modalidad de soporte ventilatorio, complicaciones asociadas al SDR como DAP, neumotórax por ruptura alveolar, septicemia, hemorragia pulmonar, apnea, hemorragia intracraneana, retinopatía del prematuro y displasia broncopulmonar (DBP) de acuerdo al diagnóstico médico registrado en ficha clínica según criterio diagnóstico de guías clínicas y resolución de la condición neonatal (sano, enfermo, trasladado o fallecido).

\section{Evaluación:}

Se revisó el libro de ingresos de la Unidad de Neonatología comprendiendo el período 1 de Enero 2014 hasta 31 Diciembre de 2014, obteniéndose el número de ficha clínica de los $\mathrm{RN}$ con el diagnóstico de SDR. La recopilación de los datos fue mediante el análisis de las fichas clínicas por las propias investigadoras. El instrumento de recolección de datos fue una planilla previamente codificada con las variables estudiadas.

Registro de información y análisis estadístico:

Los datos obtenidos en el instrumento de recolección de datos fueron recopilados en una planilla Excel, en donde se calculó para las variables cualitativas frecuencia y porcentaje y para variables cuantitativas la media, moda, mediana, desviación estándar y rango utilizando el paquete estadístico STATA 12.0.

\section{Limitaciones:}

Las limitaciones fueron los sesgos de información, debido a la ilegibilidad de la ficha clínica y la omisión, es por esto que las variables que superaron el 35\% de omisión, no fueron incluidas en los resultados. Además, no se pudieron obtener 29 fichas clínicas de neonatos con el diagnóstico de SDR descrito en el libro de ingresos de la Unidad de Neonatología puesto que estaban bajo auditoría. 
Prevalencia de etiologías del Síndrome de Dificultad Respiratoria del Recién Nacido. Perfil materno y neonatal en centro Neonatal \begin{tabular}{l|l} 
[Mat. Actual. 2020] Nro. 1: 07-16 & $\begin{array}{l}\text { ISSN: 2452-5820 } \\
\text { DOI: 10.22370/rev.mat.1.2020.2247 }\end{array}$
\end{tabular}

\section{Consideraciones éticas:}

Se consideró los principios de beneficencia y de no maleficencia. Este estudio no transgrede los derechos individuales de los participantes, puesto que se resguarda su identidad ya que a cada ficha se le asignó un código para asegurar la confidencialidad del dato. Este protocolo fue presentado a las autoridades del Hospital quienes autorizaron la revisión de las unidades de análisis.

\section{RESULTADOS}

La prevalencia del SDR en este centro Hospitalario correspondió a un 2,7\%.

Respecto a las características maternas destacó una mediana de edad de 27 años con un rango de 15 - 43 años y desviación estándar de 6,34. Con respecto a las características sociodemográficas, un $35,46 \%$ presenta enseñanza media completa seguido de un 12,06\% de media incompleta. La ocupación corresponde a un $38,3 \%$ dueña de casa y un $25,53 \%$ activa. Un $32,6 \%$ convivía, mientras que las casadas un $14,18 \%$; respecto de la previsión social un 53,9\% de las mujeres presentaban una previsión FONASA A y B. En relación a la comuna de residencia un $46,1 \%$ residía en Maipú y un 24,82\% en Santiago, la nacionalidad correspondía un $72,34 \%$ chilena y $11,35 \%$ a extranjeras. El estado nutricional, el sobrepeso y la obesidad se presentó en un 58,16\%. Por otro lado, respecto de los hábitos tanto el alcohol, tabaco y drogas, fueron consumidos durante el embarazo en un $1,42 \%, 4,96 \%$ y $3,55 \%$ respectivamente. Finalmente, un $61,7 \%$ presentó algún episodio de hospitalización en la unidad de Alto Riesgo Obstétrico durante su embarazo, del total de las madres un $83 \%$ presentó alguna patología durante la gestación de las cuales las más prevalentes fueron RPM 16,1\%, Amenaza de Parto Pretérmino 12,2\%, Preeclampsia 12,2\%, RCIU $11,1 \%$, diabetes gestacional 7,2\% e infecciones urinarias $6,1 \%$.

$\mathrm{Al}$ analizar la historia obstétrica, las madres primigestas correspondían a un $37,59 \%$, y un $25,53 \%$ secundigestas, un $43,3 \%$ eran nulíparas y un $19,9 \%$ presentó el antecedente de aborto. Con respecto al número de fetos en el embarazo actual un 84,39\% presentó feto único, 2 fetos en un 13,48\% y 3 fetos en un $2,13 \%$.
Con respecto a los datos perinatales, el tipo de ruptura de membranas fue 56,03\% intraoperatoria, rotura artificial $14,18 \%$ y rotura espontánea en un 28,37\%; respecto a las horas de membranas rotas, el intervalo de menos de 6 horas presentó un 73,05\% y mayor o igual a 24 horas un 16,31\%; según protocolo del servicio hospitalario, a las 6 horas de membranas rotas comienza la administración de antibioticoterapia, por lo que, de las madres que tuvieron mayor o igual a 6 horas de membranas rotas el 81,3\% recibió antibioticoterapia completa. El líquido amniótico tuvo como característica ser claro en un $84,4 \%$, con meconio en un $9,22 \%$, con sangre $4,96 \%$ y purulento en un $1,42 \%$. El tipo de parto fue preferentemente vía alta, destacando que un $55,3 \%$ correspondió a cesárea de urgencia y un $14,2 \%$ cesárea electiva versus un $28,4 \%$ que fue por parto vaginal, independiente del tipo de parto un $61 \%$ no presentó trabajo de parto.

La mediana de edad gestacional correspondió a 34 semanas, con un rango de 25 - 41 semanas y desviación estándar de 3,61. Al analizar según rangos de edad, se observó que el 33,3\% correspondían a recién nacidos pretérmino tardío (34 - 36 semanas), 22,7\% fueron prematuros moderados (32- 33 semanas), $18,4 \%$ fueron prematuros severos (28 - 31 semanas), $4,3 \%$ fueron prematuros extremos (menor de 28 semanas) y un 21,3\% fueron considerados de término. La administración de corticoides prenatales hasta las 34 semanas de edad gestacional, se observó que un 72,4\% recibió dosis completa, un 22,4\% incompleta, y un 5.2\% no recibió corticoterapia.

Respecto de las características neonatales, el sexo masculino representó el $52,5 \%$, versus un $47,5 \%$ femenino, un $74,5 \%$ de los RN presentó una adaptación cardiorrespiratoria normal al minuto y un $94,3 \%$ a los cinco minutos. La mediana del peso fue de 2080 gramos con un rango de 451 - 4430 gramos y desviación estándar de 890,14; la mediana de la talla fue 44 centímetros con un rango de 27 - 53 centímetros y desviación estándar de 5,47. Clasificándose en un $63,8 \%$ como adecuado y $31,91 \%$ pequeño para la edad gestacional. Un 46,1\% requirió algún tipo de intervención en reanimación neonatal y solo un 5\% presentó asfixia perinatal. En relación al momento de ocurrencia de SDR, se presentó en un 97,87\% al ingreso y un 2,13\% durante su estadía hospitalaria. 
Prevalencia de etiologías del Síndrome de Dificultad Respiratoria del Recién Nacido. Perfil materno y neonatal en centro Neonatal

La etiología más frecuente del SDR correspondió a la Taquipnea Transitoria con un $58,16 \%$ seguido de EMH con un 27,66\%, BNM con 9,22\% y SAM 2,13\% (Figura $\mathrm{N}^{\circ} 1$ ). De los RN afectados con EMH un 89,7\% recibió administración de surfactante, de estos el 100\% recibió surfactante de tipo natural, con una dosis cuya mediana fue de $4 \mathrm{ml}$ con un rango de 4 - $15 \mathrm{ml}$ y la cantidad de veces administrados corresponden a una mediana de 1 vez con un rango de 1 - 3 veces. Del total de RN un 72,3\% recibió administración de antibióticos con una mediana de 7 días y rango de 1 - 23 días. Solo un 4,5\% de los RN necesitó la administración de drogas vasoactivas, la cantidad de veces administradas corresponden a una mediana de 3 veces con un rango de 1 - 4 veces. Un 28,4\% recibió tratamiento para apnea con una mediana de 15 días y rango de $1-73$ días. Un 50\% de los RN con DAP recibió tratamiento farmacológico para esta patología, y de estos neonatos tratados solo uno requirió resolución quirúrgica.

Un $84,4 \%$ tuvo la necesidad de oxigenoterapia, con una mediana de 3 días con rango de 1 a 104 días. Con respecto a los parámetros ventilatorios y cardiorrespiratorios (Tabla $\mathrm{N}^{\circ} 1$ ), a las 6 y a las 24 horas de vida, la modalidad ventilatoria más frecuente fue CPAP con un $39,2 \%$ y $21,9 \%$; con una mediana de fracción inspirada de oxígeno (FiO2) de 24\% y $21 \%$ con un rango de 21 - 100\%; la saturación de oxígeno (SatO2) se presentó con una mediana de $96 \%$ en ambos períodos con un rango de $82 \%$ - $100 \%$ y $84 \%$ - 100\% respectivamente. Con respecto a los 7 y 14 días de vida, la modalidad ventilatoria más frecuente fue naricera con un $18,2 \%$ y $22,4 \%$, con una mediana de $\mathrm{FiO} 2$ de $21 \%$ con un rango de $21-100 \%$ en ambos períodos; la SatO2 se presentó con una mediana de $97 \%$ en ambos períodos con un rango de $89 \%$ - $100 \%$ y $90 \%-100 \%$. Al alta hospitalaria un $86,6 \%$ se fue sin soporte ventilatorio versus un $13,4 \%$ con naricera, con una mediana de $\mathrm{FiO} 2$ de $21 \%$ con un rango de 21$100 \%$; la SatO2 se presentó con una mediana de $98 \%$ con un rango de $90 \%-100 \%$.

Con respecto a los días de hospitalización el rango fue de 1 -104 días, con un promedio de 26,1, moda 7, mediana 17 y desviación estándar de 25,54. Un 43,3\% presentó alguna complicación asociada al $\mathrm{SDR}$, dentro de estas las que se presentaron en un mayor porcentaje fueron el DAP con un $25,9 \%$, DBP con un $22,2 \%$ y hemorragia intracraneana $14,81 \%$. \begin{tabular}{l|l} 
[Mat. Actual. 2020] Nro. 1: 07-16 & $\begin{array}{l}\text { ISSN: 2452-5820 } \\
\text { DOI: 10.22370/rev.mat.1.2020.2247 }\end{array}$
\end{tabular}

Finalmente, la resolución del cuadro fue en un $62,41 \%$ RN dados de alta con el diagnóstico de sanos, un 27,66\% fue dado de alta con el diagnóstico de alguna patología asociada al SDR, un 3,55\% de los recién nacidos fallecieron por causa asociada con el SDR y un $6,38 \%$ fue trasladado a otro hospital.

\section{TABLAS Y FIGURAS}

Tabla $\mathrm{N}^{0} 1$. Parámetros cardiorrespiratorios y soporte ventilatorio/ Cardiorespiratory parameters and ventilatory support.

\begin{tabular}{|c|c|c|c|c|c|}
\hline & Promedio & Moda & Mediana & $\begin{array}{c}\text { Desv. } \\
\text { estándar }\end{array}$ & Rango \\
\hline $\begin{array}{l}\text { Fracción inspirada } \\
\text { de oxígeno a las } 6 \\
\text { horas de vida del } \\
\text { RN (\%) }\end{array}$ & 30,4 & 21 & 24 & 17,88 & $21-100$ \\
\hline $\begin{array}{l}\text { Fracción inspirada } \\
\text { de oxígeno a las } 24 \\
\text { horas de vida del } \\
\text { RN (\%) }\end{array}$ & 27,42 & 21 & 21 & 13,9 & $21-100$ \\
\hline $\begin{array}{l}\text { Fracción inspirada } \\
\text { de oxígeno a los } \\
7 \text { días de vida del } \\
\text { RN (\%) }\end{array}$ & 23,57 & 21 & 21 & 11,75 & $21-100$ \\
\hline $\begin{array}{l}\text { Fracción inspirada } \\
\text { de oxígeno a los } \\
14 \text { días de vida del } \\
\text { RN (\%) }\end{array}$ & 23,86 & 21 & 21 & 13,09 & $21-100$ \\
\hline \multirow[t]{2}{*}{$\begin{array}{l}\text { Fracción inspirada } \\
\text { de oxígeno al alta } \\
\text { del RN (\%) }\end{array}$} & 22,38 & 21 & 21 & 7,937 & $21-100$ \\
\hline & Promedio & Moda & Mediana & $\begin{array}{c}\text { Desv. } \\
\text { estándar }\end{array}$ & Rango \\
\hline $\begin{array}{l}\text { Saturación a las } 6 \\
\text { horas de vida del } \\
\text { RN (\%) }\end{array}$ & 95,97 & 98 & 96 & 2,79 & $82-100$ \\
\hline $\begin{array}{l}\text { Saturación a las } \\
24 \text { horas de vida } \\
\text { del RN (\%) }\end{array}$ & 95,62 & 96 & 96 & 3,10 & $84-100$ \\
\hline $\begin{array}{l}\text { Saturación a los } \\
7 \text { días de vida del } \\
\text { RN (\%) }\end{array}$ & 96,71 & 96 & 97 & 2,43 & $89-100$ \\
\hline $\begin{array}{l}\text { Saturación a los } \\
14 \text { días de vida del } \\
\text { RN (\%) }\end{array}$ & 96.59 & 98 & 97 & 2,57 & $90-100$ \\
\hline $\begin{array}{l}\text { Saturación al alta } \\
\text { del RN (\%) }\end{array}$ & 97,81 & 100 & 98 & 3,52 & $90-100$ \\
\hline
\end{tabular}


Prevalencia de etiologías del Síndrome de Dificultad Respiratoria del Recién Nacido. Perfil materno y neonatal en centro Neonatal

\begin{tabular}{|c|c|c|c|c|c|}
\hline & Promedio & Moda & Mediana & $\begin{array}{c}\text { Desv. } \\
\text { estándar }\end{array}$ & Rango \\
\hline $\begin{array}{l}\text { Frecuencia } \\
\text { cardíaca a las } \\
6 \text { horas de vida } \\
\text { del RN (1/min) }\end{array}$ & 142,26 & 150 & 142 & 14,34 & $100-180$ \\
\hline $\begin{array}{l}\text { Frecuencia } \\
\text { cardíaca a las } \\
24 \text { horas de vida } \\
\text { del RN (l/min) }\end{array}$ & 142,57 & 150 & 142 & 13,48 & $109-182$ \\
\hline $\begin{array}{l}\text { Frecuencia } \\
\text { cardíaca a los } 7 \\
\text { días de vida del } \\
\operatorname{RN}(1 / \mathrm{min})\end{array}$ & 151,27 & 170 & 151,5 & 16,84 & $110-203$ \\
\hline $\begin{array}{l}\text { Frecuencia } \\
\text { cardíaca a los } 14 \\
\text { días de vida del } \\
\operatorname{RN}(1 / \mathrm{min})\end{array}$ & 154,25 & 154 & 153 & 14,22 & $122-195$ \\
\hline \multirow[t]{2}{*}{$\begin{array}{l}\text { Frecuencia car- } \\
\text { díaca al alta del } \\
\operatorname{RN}(1 / \mathrm{min})\end{array}$} & 149,83 & 160 & 148 & 13,81 & $117-182$ \\
\hline & Promedio & Moda & Mediana & $\begin{array}{c}\text { Desv. } \\
\text { estándar }\end{array}$ & Rango \\
\hline $\begin{array}{l}\text { Frecuencia } \\
\text { respiratoria a las } \\
6 \text { horas de vida } \\
\text { del RN (r/min) }\end{array}$ & 58,32 & 60 & 56 & 10,24 & $32-86$ \\
\hline $\begin{array}{l}\text { Frecuencia } \\
\text { respiratoria a las } \\
24 \text { horas de vida } \\
\text { del RN (r/min) }\end{array}$ & 60,15 & 60 & 59,5 & 11,72 & $32-96$ \\
\hline $\begin{array}{l}\text { Frecuencia res- } \\
\text { piratoria a los } 7 \\
\text { días de vida del } \\
\mathrm{RN}(\mathrm{r} / \mathrm{min})\end{array}$ & 54,48 & 56 & 54 & 8,66 & $38-83$ \\
\hline $\begin{array}{l}\text { Frecuencia res- } \\
\text { piratoria a los } 14 \\
\text { días de vida del } \\
\text { RN (r/min) }\end{array}$ & 54,17 & 60 & 53 & 9,47 & $38-83$ \\
\hline $\begin{array}{l}\text { Frecuencia res- } \\
\text { piratoria al alta } \\
\text { del RN (r/min) }\end{array}$ & 52 & 52 & 52 & 5,68 & $40-60$ \\
\hline
\end{tabular}

\begin{tabular}{lcc}
$\begin{array}{l}\text { Modalidad ventilatoria a las } \mathbf{6} \text { horas de } \\
\text { vida del RN }\end{array}$ & (n=141) & \% \\
\hline Ventilación Mecánica & 13 & 10,00 \\
$\begin{array}{l}\text { Presión Positiva Continua en la Vía Aérea } \\
\text { (CPAP) }\end{array}$ & 51 & 39,23 \\
Ventilación de Alta Frecuencia Oscilatoria & 4 & 3,08 \\
(VAFO) & & \\
Incubadora & 8 & 6,15 \\
Hood-Halo & 1 & 0,77 \\
Naricera & 14 & 10,77 \\
Sin soporte ventilatorio & 39 & 30,00 \\
\hline
\end{tabular}

\begin{tabular}{l|l} 
[Mat. Actual. 2020] Nro. 1: 07-16 & $\begin{array}{l}\text { ISSN: 2452-5820 } \\
\text { DOI: 10.22370/rev.mat.1.2020.2247 }\end{array}$
\end{tabular}

\begin{tabular}{|c|c|c|}
\hline $\begin{array}{l}\text { Modalidad ventilatoria a las } 24 \text { horas de } \\
\text { vida del RN }\end{array}$ & $(\mathrm{n}=141)$ & $\%$ \\
\hline Ventilación Mecánica & 13 & 10,16 \\
\hline $\begin{array}{l}\text { Presión Positiva Continua en la Vía Aérea } \\
\text { (CPAP) }\end{array}$ & 28 & 21,88 \\
\hline $\begin{array}{l}\text { Ventilación de Alta Frecuencia Oscilatoria } \\
\text { (VAFO) }\end{array}$ & 3 & 2,34 \\
\hline Incubadora & 4 & 3,13 \\
\hline Hood-Halo & 1 & 0,78 \\
\hline Naricera & 17 & 13,28 \\
\hline Oxígeno a flujo libre & 1 & 0,78 \\
\hline Sin soporte ventilatorio & 69 & 47,66 \\
\hline $\begin{array}{l}\text { Modalidad ventilatoria a los } 7 \text { días de } \\
\text { vida del RN }\end{array}$ & $(\mathrm{n}=141)$ & $\%$ \\
\hline Ventilación Mecánica & 2 & 2,02 \\
\hline $\begin{array}{l}\text { Presión Positiva Continua en la Vía Aérea } \\
\text { (CPAP) }\end{array}$ & 6 & 6,06 \\
\hline Naricera & 18 & 18,18 \\
\hline Oxígeno a flujo libre & 1 & 1,01 \\
\hline Sin soporte & 72 & 72,73 \\
\hline $\begin{array}{l}\text { Modalidad ventilatoria a los } 14 \text { días de } \\
\text { vida del RN }\end{array}$ & $(\mathrm{n}=141)$ & $\%$ \\
\hline $\begin{array}{l}\text { Presión Positiva Continua en la Vía Aérea } \\
\text { (CPAP) }\end{array}$ & 3 & 3,95 \\
\hline Naricera & 17 & 22,37 \\
\hline Oxígeno a flujo libre & 1 & 1,32 \\
\hline Sin soporte & 55 & 72,73 \\
\hline Modalidad ventilatoria al alta del RN & $(\mathrm{n}=127)$ & $\%$ \\
\hline Naricera & 17 & 13,39 \\
\hline Sin soporte & 110 & 86,61 \\
\hline
\end{tabular}

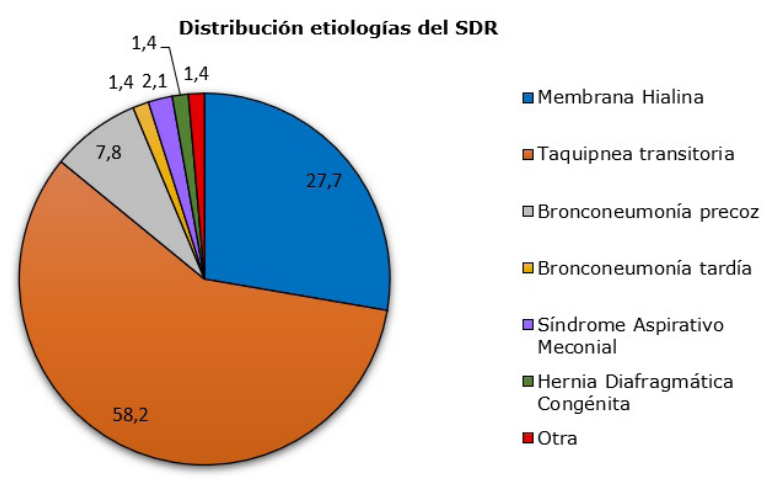

Figura $\mathrm{N}^{\circ} 1$. Distribución etiologías del Síndrome de Dificultad Respiratoria (SDR) / Distribution of respiratory distress syndrome (RDS) etiologies. 
Prevalencia de etiologías del Síndrome de Dificultad Respiratoria del Recién Nacido. Perfil materno y neonatal en centro Neonatal

\section{DISCUSIÓN}

La prevalencia del SDR en el centro hospitalario correspondió al 2,7\%, casi triplicando los datos reportados por la Guía Clínica Nacional del SDR del año 2011. Acerca de las etiologías, la más prevalente correspondió a Taquipnea Transitoria con un 58,2\%. Su principal factor de riesgo conocido es la cesárea sin trabajo de parto (12), lo cual se condice con el resultado arrojado en esta investigación en donde el tipo de parto más prevalente fue cesárea, representando un $69,5 \%$ del total de los partos. Estos resultados llevan a reflexionar con respecto a lo que sucede a nivel país en donde en promedio existe un 37,7\% de cesáreas, siendo Chile el tercer país con la tasa más alta de esta (27), correspondiendo un 37\% de cesáreas en el sistema público y $66 \%$ en el privado (28). Estos datos se contraponen con lo declarado por la OMS que recomienda que esta cifra no debería sobrepasar el $15 \% 29$, sin embargo, en este centro hospitalario en el año 2014 el porcentaje total de cesáreas representa el $31,7 \%$, los partos vaginales un $64,4 \%$ y en un 3,9\% fórceps. Lo anterior invita a plantearse: ¿sería posible que en nuestro país se pueda reducir la alta incidencia de cesáreas sin afectar en la mortalidad materna y perinatal?

La EMH tiene una prevalencia del $40 \%$ bajo las 34 semanas de edad gestacional (2) y en este estudio esta patología se presentó en un 40,8\% de los niños menores de 34 semanas, coincidiendo con lo manifestado en la literatura. De estos RN menores de 34 semanas un $94,8 \%$ recibió terapia con corticoide completa e incompleta, de los cuales solo un $37,5 \%$ presentó la enfermedad, lo que se condice con la evidencia actual que sugiere una reducción principalmente en mortalidad, SDR, hemorragia intraventricular y enterocolitis necrotizante aún si el tratamiento se ha iniciado antes de 24 horas del parto (30).

Existen estudios que relacionan características maternas con SDR, en donde se señala la edad materna mayor a 40 años, patologías como hipertensión, diabetes, anemia, metrorragia, oligohidramnios, RPM, corioamnionitis, RCIU y condiciones como el embarazo múltiple, cesárea con y sin trabajo de parto y asfixia perinatal (31). En este estudio destaca una mediana de edad materna 27 años y la presencia de patologías como Preeclampsia, Amenaza Parto Pretérmino, RPM, RCIU, ITU, Placenta Previa, \begin{tabular}{l|l} 
[Mat. Actual. 2020] Nro. 1: 07-16 & $\begin{array}{l}\text { ISSN: 2452-5820 } \\
\text { DOI: 10.22370/rev.mat.1.2020.2247 }\end{array}$
\end{tabular}

las cuales concuerdan con los datos aportados por el estudio antes mencionado, sin embargo, existen divergencias con respecto al embarazo múltiple, ya que el antecedente de embarazo simple fue el más prevalente en nuestro estudio. Por otra parte, cabe destacar que el mayor porcentaje correspondía a mujeres chilenas, dueñas de casa con un estado nutricional de sobrepeso y obesidad. Es por esto que se hace imprescindible focalizar la atención en las mujeres con estas características para poder reducir al máximo los factores de riesgos asociados al desarrollo de este cuadro.

En relación al RN, se realiza un seguimiento del cuadro considerando sus necesidades respiratorias según cada etiología, es así como en la TT un 31,7\% necesitó CPAP, en la EMH un 53,8\% requirió CPAP y un 25,6\% Ventilación Mecánica Convencional y finalmente de los RN con BRN un 30,8\% necesitó CPAP, evidenciándose la necesidad de oxigenoterapia en un gran porcentaje de los $\mathrm{RN}$, teniendo en cuenta que esta terapia no es inocua, siendo sus posibles efectos adversos y/o complicaciones la retención de $\mathrm{CO} 2$, atelectasias, barotrauma, retinopatía, enfisema intersticial, parálisis mucociliar y activación de mastocitos (32).

Existen riesgos asociados a los sistemas mecánicos, cuando se presentan problemas con válvulas, mangueras, fuente de gases, conexiones; asociadas a la vía aérea artificial: mal posición u obstrucción del tubo, extubación accidental, compromiso de los reflejos de la vía aérea y secuelas laringotraqueales, infecciones de las vías respiratorias; lesiones por barotrauma (enfisema intersticial alveolar, enfisema subcutáneo, pneumomediastino, pneumoperitoneo, neumotórax) y volutrauma (33), por lo que es importante que el profesional a cargo del neonato tenga un adecuado manejo de matronería para disminuir los riesgos asociados a las terapias ventilatorias.

Es relevante considerar que dependiendo de la edad gestacional existe una tendencia a ciertas etiologías, como podemos observar en este estudio, en donde la EMH es más frecuente en los RN menores de 28 semanas y entre 28 - 32 semanas con un porcentaje de $56,4 \%$; la TT es más frecuente entre 32 - 40 semanas de edad gestacional con un porcentaje de $89,1 \%$ y la BRN se presenta con mayor frecuencia entre las 37 - 40 semanas de edad gestacional con un porcentaje de $45,5 \%$, lo que hace necesario con- 
Prevalencia de etiologías del Síndrome de Dificultad Respiratoria del Recién Nacido. Perfil materno y neonatal en centro Neonatal

siderar este antecedente a la hora de plantearse un posible diagnóstico y el tratamiento adecuado para el RN .

En esta investigación se evidencia que un 3,2\% de los RN afectados por alguna patología respiratoria falleció, las etiologías que presentaron estos $\mathrm{RN}$ fueron en un 60\% EMH, 20\% HDC y el 20\% restante falleció debido a causas no relacionadas con SDR.

Finalmente, con respecto a las limitaciones de nuestro estudio de trabajo hubo falta de registro completo de las variables de interés en algunas fichas clínicas, siendo estas las características maternas: nivel educacional en un 34,04\%, ocupación 31,21\%, estado civil $34,05 \%$, previsión social $6,38 \%$, comuna de residencia $8,51 \%$, nacionalidad $16,31 \%$, estado nutricional $17,02 \%$, patologías maternas $6,38 \%$, tipo de ruptura de membranas $1,42 \%$, tiempo de membranas rotas en un $4,25 \%$ y terapias administradas a los neonatos y parámetros cardiorrespiratorios teniendo un máximo de $4 \%$ de omisión.

Es por esto que como equipo de investigación sugiere replicar este estudio en otros centros hospitalarios del país en donde se pueda contar con todos los datos necesarios para la investigación y poder evidenciar si los datos obtenidos en este estudio son similares en otros centros.

En conclusión, observamos un incremento de la prevalencia del SDR en el servicio de salud estudiado, siendo la etiología más prevalente de este la Taquipnea Transitoria, por lo que es relevante diseñar programas de prevención y futuras estrategias capaces de reducir las tasas de SDR, considerando el impacto en la morbimortalidad neonatal y los costos asociados al ingreso a las unidades de neonatología que este síndrome implica.

\section{REFERENCIAS.}

1- Cannizzaro, C; Paladino, M. Fisiología y fisiopatología de la adaptación neonatal. Anestesia Analgesia Reanimación. 2011;24 (2): 59-74.

2- Ministerio de Salud. Guía Clínica AUGE Síndrome de Dificultad Respiratoria en el recién nacido. 2011. $2^{\text {a }}$ edición.

3- Hermansen, C; Lorah, K. Respiratory Distress in the Newborn. American Family Physician. 2007; 76 (7):987994. \begin{tabular}{l|l} 
[Mat. Actual. 2020] Nro. 1: 07-16 & ISSN: 2452-5820 \\
DOI: 10.22370/rev.mat.1.2020.2247
\end{tabular}

4- Cajina, F. Síndrome de Dificultad Respiratoria Neonatal (SDR). Nicaragua Pediátrica. 2013;1 (1): 37-40.

5- National Neonatal Perinatal Database Network: report 2002-2003. New Delhi: National Neonatology Forum of India; 2004.

6- López, C; Santamaría, C; Macías, H; Cruz, J. Dificultad respiratoria por deficiencia de surfactante en un hospital público de tercer nivel sin maternidad. Factores asociados a mortalidad. Boletín Médico del Hospital Infantil de México. 2010;67 (2): 98107.

7- Subsecretaría de Salud Pública. División Prevención y Control de Enfermedades. Departamento Ciclo Vital. Programa Salud de la Mujer. Guía Perinatal 2013.

8- Pickerd, N; Kotecha, S. Pathophysiology of respiratory distress syndrome. Paediatrics and ChildHealth. Symposium: Neonatology. 2008;19 (4): 153157.

9- Velázquez, P; Vega, G; Martínez, M. (2010). Morbilidad y mortalidad neonatal asociada a la diabetes gestacional. Revista Chilena de Obstetricia y Ginecología 2010; 75 (1): 35-41.

10- López, F; Meritano, J; Da Reprecentaçao, C; Licudis, M; Romano, A; Valenti, E. Síndrome de dificultad respiratoria neonatal: comparación entre cesárea programada y parto vaginal en un recién nacido de término. Revista del Hospital Materno Infantil Ramón Sardá. 2006;25 (3): 109-111.

11- Coto, G; López, J; Fernández, B; Álvarez, F; Ibáñez, A. Recién nacido a término con dificultad respiratoria: enfoque diagnóstico y terapéutico. Protocolos Diagnóstico Terapeúticos de la AEP: Neonatología. Asociación Española de Pediatría. 2008, pp. 285-305.

12- Bazán, G; Martínez, C; Zannota, R; Galván, O; Grasso, D; Martino, N; Bustos, R; Sosa, C. El trabajo de parto previo a la cesárea protege contra la taquipnea transitoria del recién nacido. Archivos de Pediatría del Uruguay. 2012;83 (1).

13- Baquero, H; Galindo, J. Respiración y circulación fetal y neonatal Fenómenos de adaptabilidad. Programa de educación continua en pediatría. 2006, módulo 1. pp 1-15.

14- Hillman, N; Kallapur, SG; Jobe, A. Physiology of transition from intrauterine to extrauterine life. Clin Perinatol. 2012; 39: 769-783.

15- Novoa, P. 2010. Factores de Riesgo de Bronconeumonía connatal en pacientes atendidos en el Hospital Vicente Corral Moscoso. Especialista en 
Prevalencia de etiologías del Síndrome de Dificultad Respiratoria del Recién Nacido. Perfil materno y neonatal en centro Neonatal

Pediatría. Cuenca, Ecuador. Universidad de Cuenca, Facultad de Ciencias Médicas, Postgrado de Pediatría. p.32.

16- Edwards, M; Kotecha, SJ; Kotecha, S. Respiratory Distress of the Term Newborn Infant. Pediatric Respiratory Reviews. 2013; 14: 29-37.

17- Meritano, J; Abrahan, M; Di Pietro, S; Fernández, V; Gerez, G. Síndrome de Dificultad Respiratoria asociado a líquido amniótico meconial en Recién Nacidos de término y postérmino: Incidencia, Factores de Riesgos y Morbimortalidad. Revista del Hospital Materno Infantil Ramón Sardá. 2010;29 (3): 113-119.

18- Fischer, C; Rybakowski, C; Ferdynus, C; Sagot, P; Gouyon, J. A Population-Based Study of Meconium Aspiration Syndrome in Neonates Born between 37 and 43 Weeks of Gestation. International Journal of Pediatrics Volume. 2012, Article ID 321545, 7 pages 7 .

19- Dargaville, P; Copnell, B. The epidemiology of meconium aspiration syndrome: incidence, risk factors, therapies, and outcome. Pediatrics. 2006; 117: 1712-1721.

20- Gallardo, K; Panduro, J; Camarena, E; Quintero, I; Barrios, E; Fajardo, S. Repercusiones perinatales en embarazos a término con oligohidramnios severo. Revista Médica MD. 2013;4 (4): 245-250.

21- Goire, M; Pérez, K; Álvarez, I; Regüeiferos, L; Hernández, H. Factores de riesgo del síndrome de aspiración meconial. MEDISAN. 2006;10 (3).

22- Abman, S; Baker, C; Gien, J; Mourani, P; Galambos, C. The Robyn Barst Memorial Lecture: Differences between the fetal, newborn, and adult pulmonary circulations: relevance for age-specific therapies (2013 Grover Conference series). Pulm Circ. 2014;4 (3): 424-440.

23- Cabral, J; Belik, J. Persistent pulmonary hypertension of the newborn: Recent advances in pathophysiology and treatment. J Pediatr (Rio J). 2013;89 (3): 226-242.

24- Osorio, W; Nachar, R. Hipertensión pulmonar persistente neonatal (HPPN). Guías de Práctica Clínica del Servicio de Neonatología del Hospital Luis Tisné Brousse. Disponible en: [http://gpcneonatologia.blogspot.com].

25- Rodas, S; Fariña, D. Hipertensión pulmonar persistente del recién nacido. Enfermería Neonatal. 2012;4 (13): 5-10.
[Mat. Actual. 2020] Nro. 1: 07-16 $\mid \begin{aligned} & \text { ISSN: 2452-5820 } \\ & \text { DOI: 10.22370/rev.mat.1.2020.2247 }\end{aligned}$

26- Castro, W; Labarrere, Y; González, G; Barrios, Y. Factores de riesgo del Síndrome Dificultad Respiratoria de origen pulmonar en el recién nacido. Revista Cubana de Enfermería 2007;23 (3): 1-15.

27- Gobierno de Chile, Ministerio de Salud. Panorama de Salud 2013. Informe OECD sobre Chile y comparación con países miembros. Disponible en: [http://web.minsal.cl/sites/default/files/INFORME\%20OCDE_2013_21\%2011_final.pdf].

28- Guzmán, E. Perfil epidemiológico de la cesárea en Chile en la década 2000-2010. Medwave. 2012;12 (3):1-4.

29- Tasas de cesáreas: análisis de los estimados regionales y nacionales. Rev Panam Salud Publica. 2007;21 (4): 251-251.

30- Alegría, X. Corticoides prenatales: Beneficios y riesgos asociados a su uso indiscriminado. Rev. Obstet. Ginecol. Hops. Santiago Oriente Dr. Luis Tisné Brousse. 2006;1 (1): 69-71.

31- Sun, H; Xu, F; Xiong, H; Kang, W; Bai, Q; Zhang, Y; "et. al". Characteristics of Respiratory Distress Syndrome in Infants of Different Gestational Ages. Lung. 2013; 191:425-433.

32- Sánchez, R; Peñaranda, D; Rivera, L; Mendivil, E. Cómo puede ser tóxico el oxígeno en el período neonatal. CCAP. 9 (4): 35-42.

33- Gutiérrez, F. Ventilación mecánica. Acta Méd Peruana. 2011; 28 (2): 87-104. 